\title{
Design Parameterization for Concurrent Design and Manufacturing of Mechanical Systems
}

\author{
Javier Silva and Kuang-Hua Chang* \\ Concurrent Design and Manufacturing Research Laboratory and School of Aerospace and Mechanical Engineering \\ 865 Asp Avenue, Room 201, The University of Oklahoma, Norman, OK 73019
}

Received 28 January 2001; accepted in revised form November 2001

\begin{abstract}
Design changes are frequently encountered in the product development process. The complexity of the design changes is multiplied when the product design involves multiple engineering disciplines. Very often, a simple change in one part may propagate to its neighboring parts, therefore, affects the entire product assembly. Both parts and assembly must be regenerated for a physically valid product model, at the same time, the regenerated product model must meet designer's expectations.

When a product is being developed in a Concurrent Design and Manufacturing (CDM) environment, the design changes are usually implemented first by altering geometry of the product represented in computer-aided design (CAD) solid models. If the product solid model is not parameterized properly, the changes in geometry often lead to invalid parts or assembly. At the part level, the changes may yield a solid model with invalid geometric features if it is not properly parameterized. In this case, the entire product assembly is in vain. Even when individual parts of the product are regenerated correctly, parts may still penetrate to their neighboring parts or leave excessive gaps among them, if the solid model is not properly parameterized at the assembly level.

In this paper, solid modeling and assembly techniques implemented in two major CAD tools, Pro/ENGINEER and SolidWorks, will be discussed. A set of guidelines will be proposed for the designers to parameterize the solid models in order to capture the design intents more effectively in the product virtual mockup. These guidelines at both part and assembly levels will support designers to successfully conduct product design in the CDM environment.

A number of examples, including a slider-crank mechanism and its crankshaft, a single-piston airplane engine and its components, as well as a number of simpler parts are presented to illustrate and demonstrate the parameterization method and guidelines proposed for both Pro/ENGINEER and SolidWorks. Note that in this paper parts and assembly are created in respective CAD tools. Issues of solid model translations between CAD systems will not be addressed.
\end{abstract}

Key Words: design parameterization, design intent, concurrent engineering, virtual prototyping, rapid prototyping, design trade-off.

\section{Introduction}

After significant research and development in the past two decades, the feature-based and parametric modeling techniques have become a reality [1,2]. The featurebased and parametric modeling techniques have been widely adopted in the mainstream CAD tools, such as Pro/ENGINEER, SolidWorks, SolidEdge, Unigraphics, CATIA, I-DEAS, and even Mechanical Desktop of AutoCAD. With such techniques, designers are able to create parts through geometric features, and assemble parts or subassemblies for the product digital mockup in the CAD environment. In addition, the designer will be able to define design variables by relating dimensions of the part features and imposing assembly constraints between parts to parameterize the product model through the parametric modeling technique.

*Author to whom correspondence should be addressed

E-mail: khchang@ou.edu
With the parameterized product model, the designer can make a design change simply by changing design variable values and asking the CAD software to automatically regenerate the parts that are affected by the change, hence regenerating the entire assembly.

For example, the bore diameter of an engine case is defined as the design variable, as shown in Figure 1a. When the diameter is changed from $1.2^{\prime \prime}$ to $1.6^{\prime \prime}$, the engine case is regenerated by properly updating geometric features that are affected by the change. As shown in Figure 1b, the engine case becomes wider and the distance between the two exhaust manifolds is larger, just to name a few. At the same time, the change propagates to other parts in the assembly, including piston, piston pin, cylinder head, cylinder sleeve, cylinder fins, and crankshaft, as illustrated in Figure 1b. More importantly, the parts stay intact, maintain adequate assembly placement constraints, and the change does not induce interference nor leave excessive gaps between parts. With such parametric models, designers 


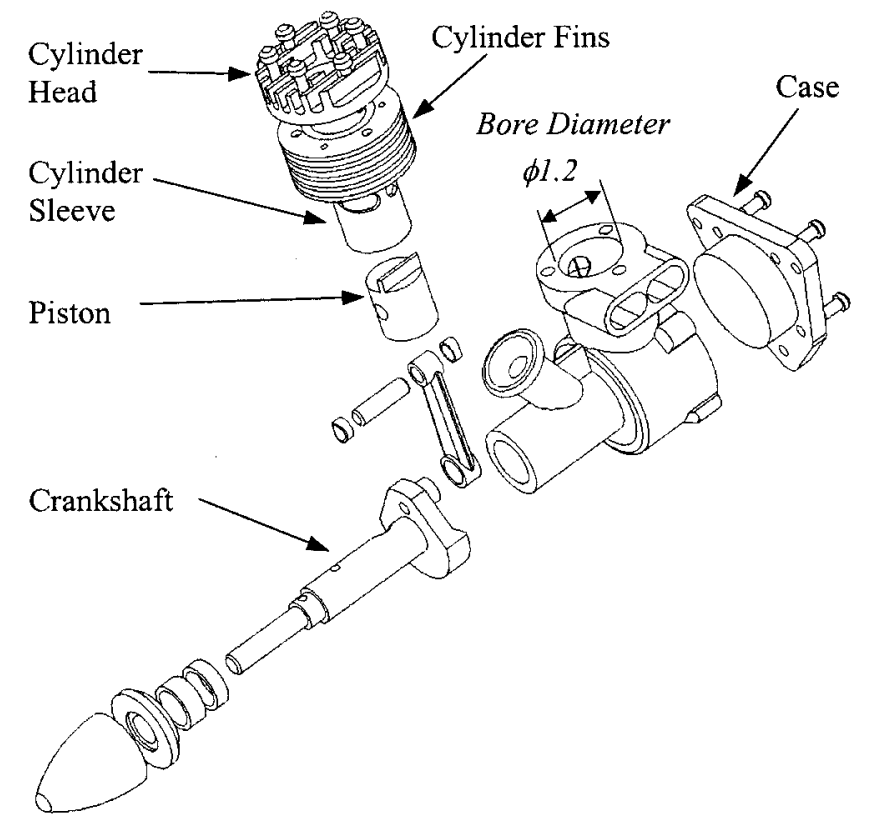

(a) Bore Diameter 1.2"

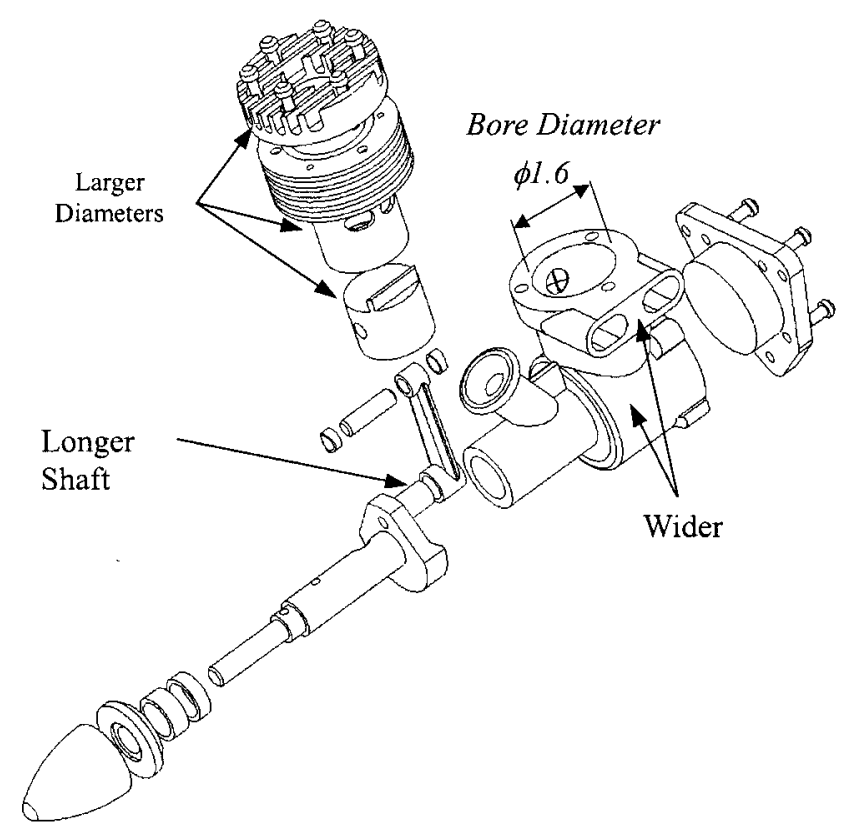

(b) Bore Diameter 1.6"

Figure 1. A single-piston engine - exploded view.

are given tremendous freedom to explore design alternatives efficiently and accurately. In addition, this parametric technology supports the cross-functional team to conduct parametric study and design tradeoffs in the CDM environment [3].

When a number of feasible design alternatives are identified, designers must make design decisions by conducting design trade-offs between product cost and performance. The CDM environment employs physics-based computational methods together with computer graphics technique to support a crossfunctional team analyzing product performance, reliability, and manufacturing cost early in the product development stage; and conducting quantitative tradeoff for design decision making. Physical prototypes of the product design are then produced using Rapid Prototyping (RP) technique primarily for design review and verification. The CDM approach holds potential for shortening the overall product development cycle, improving product quality, and reducing product cost [3].

The objectives of the paper are to investigate the parametric capabilities implemented in Pro/ENGINEER and SolidWorks, and propose a set of parameterization guidelines that support the designer to capture design intents more effectively. The parametric solid models are indispensable for the cross-functional team to conduct product design in the CDM environment.

The paper is organized as follows. The CDM paradigm and software environment are briefly discussed first. More details about CDM can be found in [3]. The product design intents that must be properly captured for product design in CDM are discussed next. Design intents capturing at part and assembly levels are discussed together with the proposed guidelines.

\section{Concurrent Design and Manufacturing (CDM) Paradigm}

The CDM paradigm consists of Virtual Prototyping (VP) for product design and RP for fabricating product physical prototypes, as shown in Figure 2. In CDM, a product design concept is first created in solid models by designers, using CAD tools. The initial product is often established based on designer's experience and legacy data of previous product lines. It is highly desirable to capture and organize the experience and legacy data to support decision-making in a discrete form in order to realize an initial concept design. The Knowledge-Based Engineering (KBE) that computerizes knowledge about certain product domain to support design engineers to arrive at a design solution is desirable to support the concept design. Moreover, a $\mathrm{KBE}$ system integrated with a CAD tool will directly generate solid model of the concept design that serves directly the down-stream design and manufacturing simulations.

With the product solid model represented in CAD, simulations for product performance, reliability, and manufacturability can be conducted. The product development task and the cross-functional team are decomposed according to disciplines and expertise. Based on a centralized CAD product model, simulation 


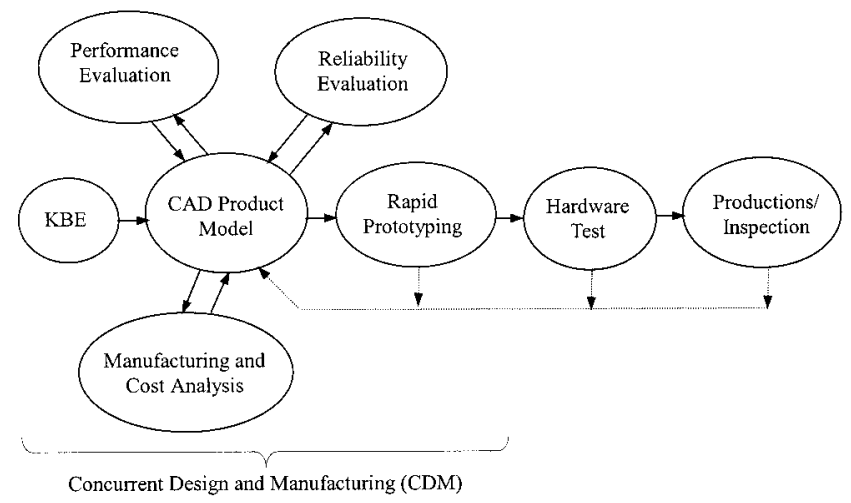

Figure 2. Concurrent design and manufacturing process.

models can be derived with proper simplifications and assumptions. However, at least one way mapping that governs changes of the CAD models to changes of simulation models must be established for rapid simulation model updates [4]. The mapping maintains consistency between CAD and simulation models throughout the product development cycle.

Product performance, reliability, and manufacturing process can then be simulated concurrently. Product performance, quality, and cost obtained from multidisciplinary simulations are brought together for review by the cross-functional team. Design variables, including geometric dimensions and material properties of the product CAD models, that significantly influence the product performance, quality, and cost are identified by the cross-functional team in the CAD product model. These essential performance, quality, and cost measures, as well as design variables constitute a product design model. With such a design model, a systematic design approach, including parametric study for concept design and trade-off study for detail design, can be conducted to improve product with a minimum number of design iterations [5].

The product designed in the virtual environment can then be fabricated using RP for product physical prototypes directly from CAD solid models, without tooling and process planning. The physical prototypes provide the cross-functional team with an opportunity for design review and verification. Design change requests made at this point can be accommodated in the CDM environment without high cost and delay.

The physics-based simulations potentially minimize the needs for product hardware tests. Due to substantial modeling and simulations performed, surprising design defects encountered during the hardware tests will not be common; thus, minimizing the feedback loop for design modifications. Moreover, production process will be smooth since manufacturing process has been planned and simulated. Potential manufacturing-related problems should have been largely addressed in earlier stage.
The essential factor for a successful product design using the CDM paradigm is that the designer properly captures the design intents in the product solid models using the feature-based parametric modeling capabilities in CAD. With such intents captured, the design team is able to explore design alternatives more effectively through parametric study and design trade-offs.

\section{Design Parameterization for Capturing Design Intents}

In the context of CDM paradigm, Design Intent (DI) is defined as a product performance measure that the designer desires to attain by changing geometric shape of parts and their placements with respect to other parts in an assembly. This design change is usually realized through change of geometric dimension values in the CAD solid model. In order to capture the DI, the product solid model must be properly parameterized.

Design intent for a single part can be captured by properly creating individual geometric features and carefully relating dimensions within or among features so that when a dimension value is changed, the geometric features affected by the change can be regenerated or rebuilt successfully. The geometric dimensions that can be changed independently to capture DIs are called Design Variables (DVs).

At the assembly level, DI is captured by imposing adequate placement constraints and relating dimensions across parts so that a change in dimension value can be propagated to all parts affected. Parts affected must be regenerated successfully, at the same time, they must maintain proper positions and orientations with respect to one another without violating any assembly placement constraints or revealing part penetration or excessive gaps. Moreover, the regenerated solid model must meet the designer's expectations. At the part level, design parameterization implies creating features and relating dimensions. At the assembly level, design parameterization involves defining placement constraints and relating dimensions across parts.

In this paper, a set of guidelines for design parameterization are proposed following two important axioms, borrowed from Suh [6]. They are:

Axiom 1: The Independence Axiom

Maintain the independence of design intents.

Axiom 2: The Information Axiom

Minimize the information contents of the design intents.

Axiom 1 implies that changing the DV values has an effect only on the referent DI. In other words, it is desirable to create uncoupled DIs whenever it 


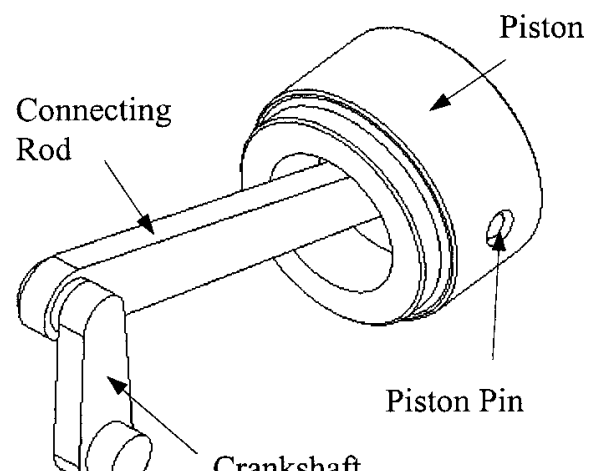

Crankshaft

(a) Unexploded View

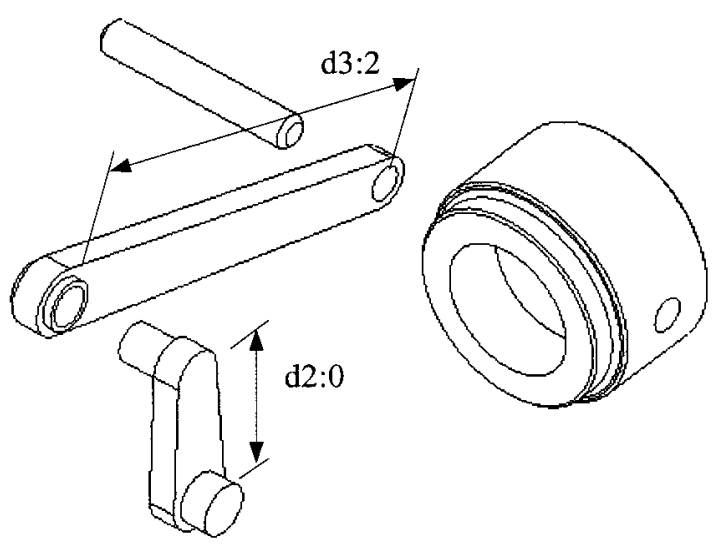

(b) Exploded View

Figure 3. The slider-crank mechanism.

is possible. Since this may not be always possible, when coupled DIs are unavoidable, trade-offs must be conducted to search for a compromised but nearoptimal design. Axiom 2 states that the amount of information (number of DVs) that is available to the designer for design changes must be minimized for DI. A simple slider-crank example shown in Figure 3 is presented to illustrate the issues involved in the design parameterization.

This slider-crank mechanism consists of four parts, crankshaft, connecting rod, piston pin, and piston. Two design intents are defined in this example: (i) horizontal velocity of the piston increases $20 \%$ when the crankshaft is driven at the same angular velocity, and (ii) weight of the mechanism reduces $30 \%$. The first intent can be captured by defining lengths of the crankshaft $(\mathrm{d} 2: 0)$ and rod $(\mathrm{d} 3: 2)$ as two DVs, as shown in Figure 3b. However, changing either one will also affect the second intent, weight of the mechanism. In this case, these two DIs are coupled.

In order to reduce the coupling effect in these two intents, additional DVs, for instance, width of the

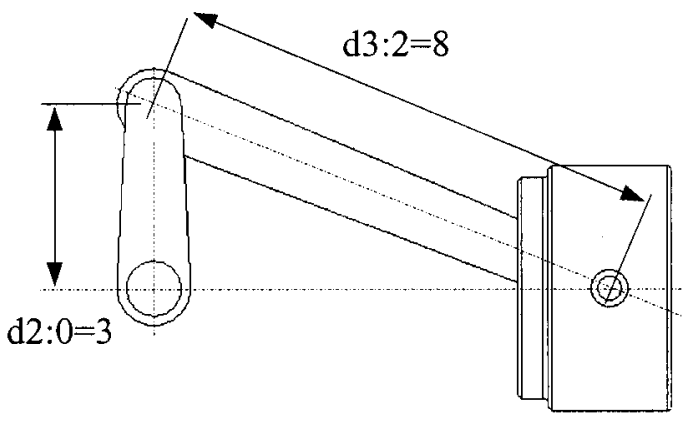

(a) Design Variables d2:0 and d3:2

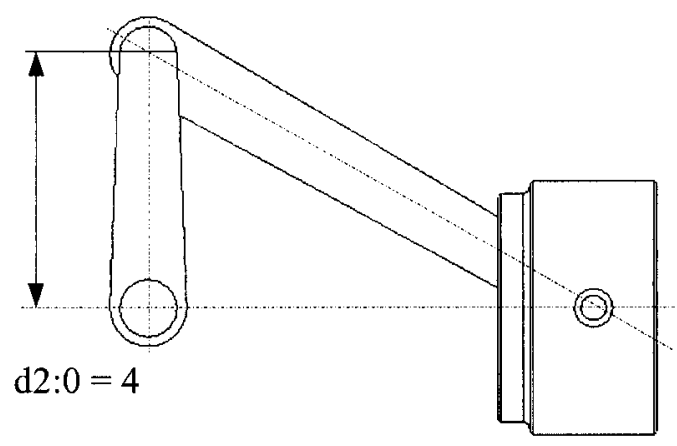

(b) d2:0 Changes to 4

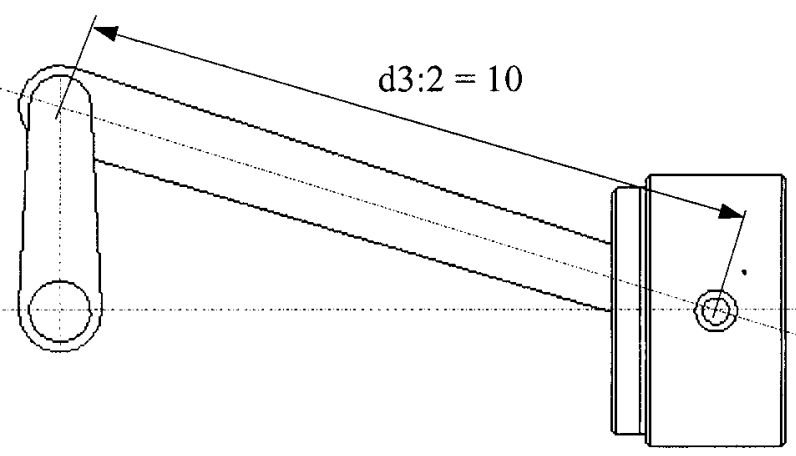

(c) d3:2 Changes to 10

Figure 4. Changes of length design variables.

crankshaft or outer diameter of the piston, can be defined to support the second intent or to compensate the effect of the first two DVs. Therefore, adding DVs for the second intent help decouple the design, therefore, better comply with Axiom 1.

In particular, it is desirable for the designer to change only the values of two DVs $\mathrm{d} 2: 0$ and $\mathrm{d} 3: 2$ while exploring design alternatives for the first DI. This is what Axiom 2 asks. When any of the two DVs is changed, the change must be propagated to the affected parts. The remaining parts must be kept unchanged, and the entire assembly must be maintained intact, as illustrated in Figure 4. 


\section{Design Parameterization at Part Level}

Design parameterization must be conducted at both part and assembly levels. At the part level of the slidercrank example, the geometry of the crankshaft or rod must be changed following certain rules that were established when the parts were created. In this section, the general modeling capabilities and modeling procedure will be discussed. Guidelines for design parameterization at the part level will be proposed.

\subsection{Solid Modeling Capabilities and Procedure}

In CAD, the solid modeling procedure usually starts with defining datum features, such as datum planes, datum coordinate systems, datum axes, etc., that serve as the references to facilitate creating the geometric features. One of the datum planes is usually chosen to sketch a 2-D profile that will be protruded to create the first (or base) 3-D feature.

In sketch, geometric entities, such as lines, arcs, splines, are drawn as vectors for a single open or closed profile that can be protruded for a solid feature or making a cut. A set of characteristics points are created for these vector entities. The profile is mainly determined by the $x$ and $y$ positions of the characteristics points. In both Pro/ENGINEER and SolidWorks, implicit constraints, such as concentric of circular arcs, parallel of lines, etc., are generated automatically when these entities are drawn. The designer may define additional dimensions and implicit constraints that fully constrains the profile. Internally, the variational modeling technique is employed to define and regenerate the profile. That is, a number of equations are created to uniquely determine the position of the characteristics points, by combining implicit constraints and dimensions.

When the first feature is protruded, parametric surfaces [2,7] that represent the boundary of the geometric feature are generated by CAD to define and display the feature. After that, the designer may create additional datum, sketches, and protrusion features; using options, such as extrusion, sweep, revolve, and blend. The designer may also cut the existing features; generate chamfers or rounds; or copy, mirror, and/or pattern the existing features; to create additional features.

When additional features are created, a Constructive Solid Geometry (CSG) tree (or model tree) is generated by CAD following the feature creation sequence. The Boolean operations are employed to union or subtract the features from the previous ones according to their definitions. At the same time, the intersection curves between boundary surfaces are calculated to evaluate the Boolean operations and display the features. This is essentially the CSG method.

Note that in general the intersection curves are approximated by interpolating a number of intersection points using B-Spline or NURB curves [2]. The evaluated geometry and topology of boundary faces, edges, and vertices are stored in CAD database for display. This is the Boundary Representation (B-Rep) method. When features are being created, the designers can define Relations in Pro/ENGINEER (or Equations in SolidWorks) to relate feature dimensions to capture DIs. In this process, independent and dependent dimensions will be created to define a one-way relation. The independent dimensions become DVs. This is so-called "unidirectional" or "procedural" parametric modeling [8].

Once all the features are created and relations are established, the part solid model is completely defined. When a design change is conducted by changing the DV values, the solid model will be regenerated in Pro/ENGINEER (or rebuilt in SolidWorks) by updating features (both datum and geometric features) following the model tree, one at a time.

Note that if the DIs are not properly captured in features and relations, the regeneration may lead to an undesirable or an invalid solid model. It is strongly recommended that the designer sketch a model build plan with details of features, dimensions, and relations, before creating any features in CAD.

\subsection{The Independence Axiom}

In the part level, Axiom 1 is addressed as follows. Perturbation of a DV must affect only its referent DI; therefore, all DIs must be uncoupled. In the part level, the independence of DIs can be imposed in the solid model through sketches and features.

\section{UNCOUPLED DESIGN}

An uncoupled design is always superior to a coupled or decoupled one. This is because that the DIs in the uncoupled design can be attended much easier, since the effect of individual DV on DI is completely separated. Moreover, an uncoupled design carries less information to the designer.

An example of the uncoupled design is shown in Figure 5a. The design of a plate with an orifice used to measure flow rates has two DIs:

DI1: position of the orifice, composed by $\mathrm{d} 0$ and $\mathrm{d} 1$; and

DI2: height of the plate, composed of $\mathrm{d}$.

Perturbation in the DV value that defines the position of the hole has no effect on the height of the plate.

\section{COUPLED DESIGN}

The same plate example may be created as a coupled design, as shown in Figure 5b. Perturbing d1 affects the height of the plate. In order for the two DIs to remain independent, they need to be referenced to a datum that 
is not a DV. Both solid models in Figures $5 \mathrm{a}$ and $5 \mathrm{~b}$ are valid; however, the solid model in Figure 5a will provide the designer with a clearer perspective on how each DV affects its own DI. Although the solid model in Figure $5 \mathrm{~b}$ is valid, it does not comply with Axiom 1, therefore, its use in the design process may render cumbersome.

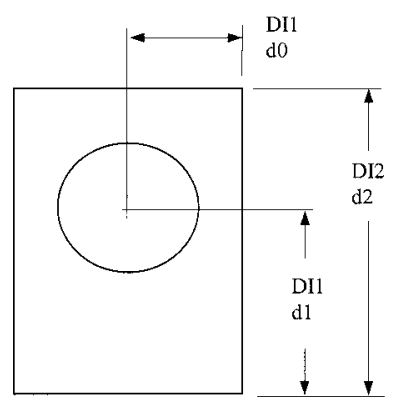

(a) Uncoupled DIs

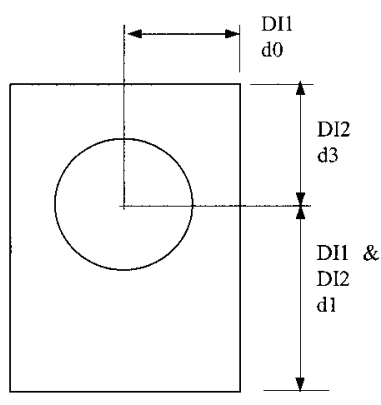

(b) Coupled DIs

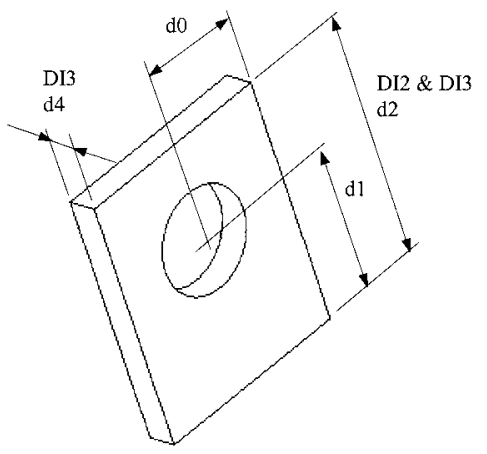

(c) Decoupled DIs

Figure 5. Various design intents.

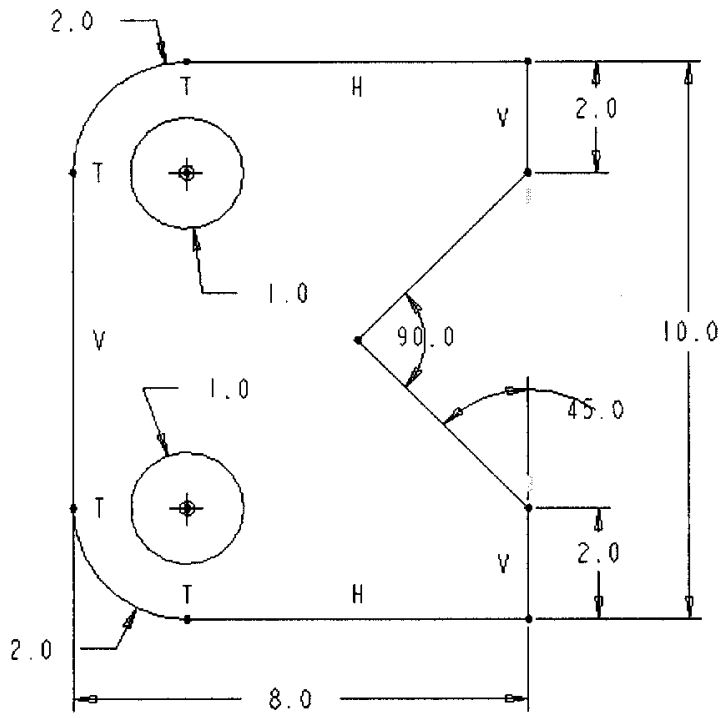

(a) Information Not Minimized

\section{DECOUPLED DESIGN}

In many design problems, it may not be always possible to define completely uncoupled DIs. A coupled design violates Axiom 1, therefore the DIs need to be made at least decoupled. For example, volume of the plate (DI3) represents a coupled design. In this case, d2 affects DI3, as illustrated in Figure 5a. Hence, DI2 and DI3 are coupled. As shown in Figure 5c, the solid model needs to be made decoupled by adding d4 to DI3: thickness of the plate.

\subsection{The Information Axiom}

The second axiom can be primarily addressed in the sketch of the geometric feature. A bracket example created in Pro/ENGINEER shown in Figure 6 is employed for illustration. The bracket profile consists of two horizontal and three vertical line segments, two perpendicular line segments, two quarter circular arcs, and two circles. By using the Intent Manager of Pro/ ENGINEER [9], the profile is fully constrained with ten dimensions, and a number of implicit constraints, including vertical, horizontal, tangent, concentric, and vertical alignment (see Figure 6a).

Note that in this profile, the information contents are not minimized. For example, one of the DIs is to keep the profile symmetric with respect to its middle horizontal line. In order to capture the symmetry DI, entities must be related. For examples, the radii of the two circular arcs, the radii of the circles, and lengths of the two vertical line segments must be changed simultaneously. Additional implicit constraints are defined to parameterize the profile in order to capture the DI. Two equal radii constraints $\mathrm{R} 1-\mathrm{R} 1$ and $\mathrm{R} 2-\mathrm{R} 2$,

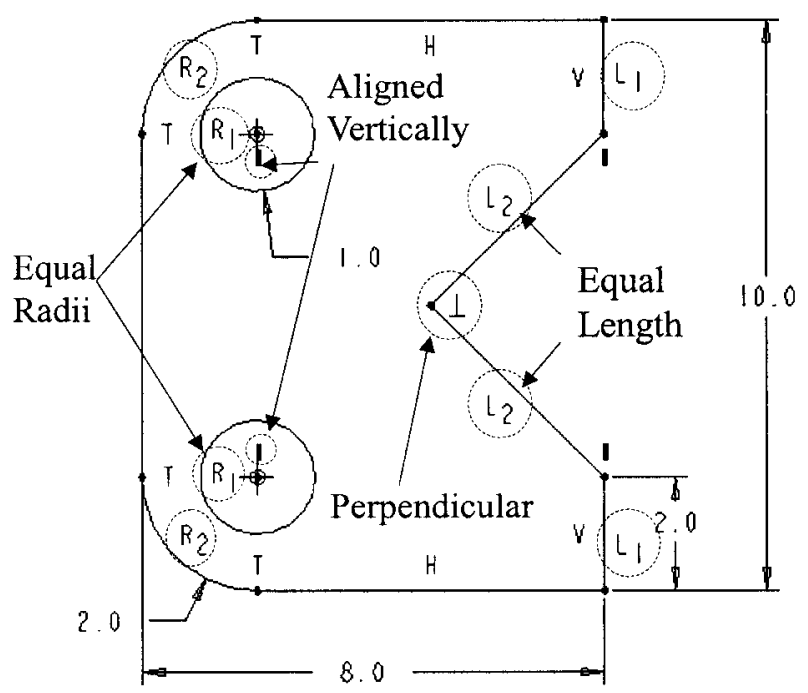

(b) Information Minimized

Figure 6. Minimization of information contents. 
two equal lengths constraints, L1-L1 and L2-L2, one vertical alignment constraint (symbol I), and a perpendicular constraint $(\perp)$ are defined. As a result, the DI is properly captured and the number of dimensions is reduced to five, as shown in Figure $6 \mathrm{~b}$. Note that the profile in Figure 6b complies much better with Axiom 2. Note that the profile shown in Figure $6 \mathrm{~b}$ can also be created in SolidWorks since similar sketching capabilities and implicit constraints are available in SolidWorks sketch mode, but without symbols.

In addition to section profile, relations or equations can be added to relate dimensions between features. When new features are created by copying, mirroring, or patterning existing features, additional dimensions may be assigned as dependent to those of the original feature in order to reduce the information contents, if it is consistent to the DI to capture.

\subsection{Crankshaft Example}

The crankshaft is created in both Pro/ENGINEER and SolidWorks in the following sequence: three default datum planes (DTM1, 2, 3), a default coordinate system $(C S 0)$, a base protrusion feature, the lower protrusion feature, and the upper protrusion feature, as illustrated in Figure 7. Note that in SolidWorks, datum planes and coordinate system are given for each part by default. The base protrusion feature is created by sketching its section profile on DTM3 and extruding 0.5 unit along the normal direction of DTM3. The sketch is drawn using two semi-circles and two straight line segments, with the dotted lines (representing DTM1 and DTM2) shown in Figure 7 as the references. With the center points of the semi-circles aligned with the references and various implicit constraints, only three dimensions are needed to completely define the sketch, i.e., radii of the semi-circles and the vertical distance between the center points. Since the minimal number of dimensions is employed for the sketch, crank length design variable can be easily captured in the base feature.

There are six characteristic points generated in the profile, as shown in Figure 8. Hence, it requires twelve independent equations to uniquely determine the positions of the characteristic points. This profile

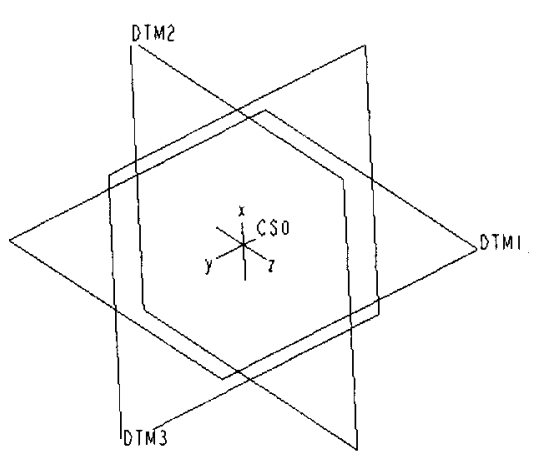

Datum Planes and Datum Coordinate System
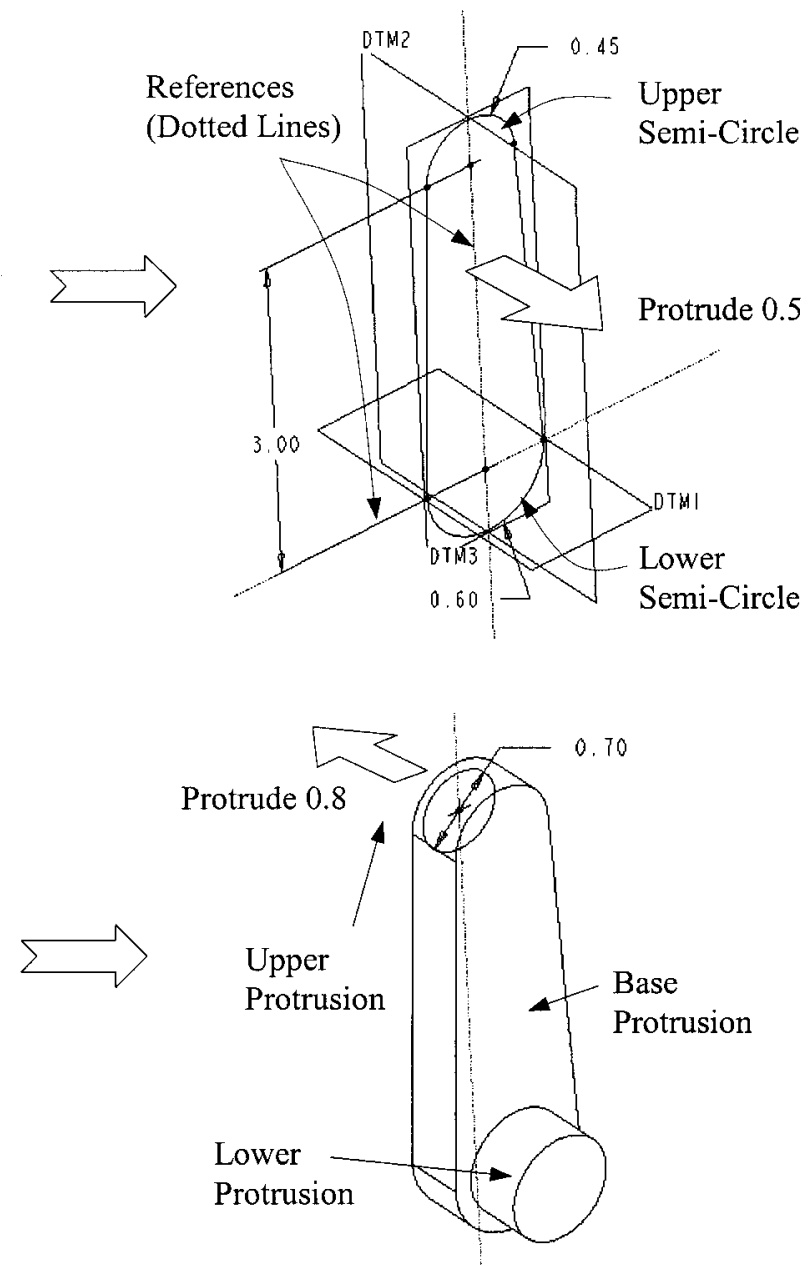

Protrude 0.5

Figure 7. Feature creation steps of the crankshaft. 
consists of six implicit constraints, as listed in Figure 8, and three dimensions. These twelve equations can be formulated by employing the implicit constraints and dimensions, as shown in Figure 8. Note that in this case, these equations are linear, hence, they can be solved by matrix operations. In general, linear equations cannot always be expected. In these cases, Newton-Raphson method is employed to solve for the positions. When a design variable is changed in the sketch, the same set of equations are solved for the new positions, hence updating the profile.

As shown in Figure 7, the lower protrusion feature is created by drawing a circle of diameter 0.9 that is concentric with that of the lower semi-circle of the base feature, and extruding 0.5. Similarly, the upper protrusion feature is created by drawing a circle of diameter 0.7 that is concentric with that of the upper semi-circle of the base feature, and extruding 0.8 in the opposite direction.

By imposing the alignment and concentric rules, the crankshaft is properly parameterized, yet the number of dimensions in the crankshaft solid model is minimized,

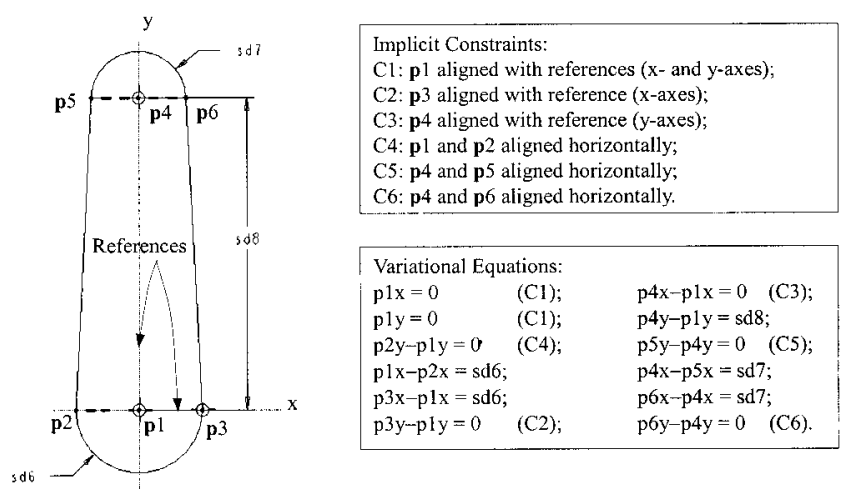

Figure 8. Variational equations for the section Profile. hence complying with Axiom 2. The change of crank length can be realized by simply modifying the dimension $\mathrm{d} 2: 0$. The base protrusion feature is updated according to its sketch shown in Figure 8. The lower protrusion feature is unchanged since it is concentric with the lower semi-circle of the base feature. The upper protrusion feature is pushed upward since it is concentric to the upper semi-circle of the base feature and the center point moves up due to the references chosen. The change is propagated to features in the crankshaft through the model tree established following the feature creation sequence and Boolean operations.

\subsection{Guidelines for Design Parameterization}

Based on the previous discussions, a set of guidelines for part parameterization are proposed in Table 1 . These guidelines are separately listed according to the two axioms and the various steps in modeling. Note that a build plan is desirable to develop before creating any features.

\section{Design Parameterization at Assembly Level}

In this section, placement constraints employed in Pro/ENGINEER and SolidWorks for assembly will be discussed first. The two axioms at the assembly level will be addressed. The slider-crank example will be used to illustrate the assembly capabilities in both CAD tools.

\subsection{Placement Constraints}

There are six degrees of freedom (dof's) for each part in space, three translations and three rotations. In Pro/ENGINEER, the first part can be assembled to

Table 1. Proposed guidelines for part parameterization.

Independence Axiom

Datum

Sketch

Solid features

Parts
A solid model should always be started with the creation of three orthogonal datum planes.

A Design Variable (DV) should never be referenced to another DV or to another geometric dimension (unless the design intent requires it). A DV should be referenced as much as possible to nonchanging features such as datum planes. or may be defined with reference to a surface, point, curve, or boundary. The depth can also be defined by a relation.

After the solid model is built, the designer should only have access to dimensions that form a DI, that is, only to DVs.
Information Axiom

Additional datum features should be referenced to the independent orthogonal datum planes.

The information content can be easily minimized by the use of alignment and symmetry. Alignment and symmetry should be used as much as possible. Relations must be added not only to minimize the information content but also to capture the DI.

Redundant and zero-valued dimensions should never be defined. These are completely useless and tend to confuse the designer when relations are being established.

The amount of information in solid features that have one or more planes of symmetry can be minimized using pattern, copy, and mirror.

Upon a design change, all the dimensions that are not DVs should be automatically updated via relations. The relations established between features need to capture the design intent. 
the assembly datum features, such as datum planes or datum coordinate systems, using mate or coordinate system placement constraints. In SolidWorks, the first part will be fixed, by aligning the part coordinate system with the default coordinate system defined in the assembly. Therefore, all six dof's of the first part will be eliminated. When the second part is brought into the assembly, there are additional six dof's for the designer to work with.

For example, the lower shaft of the crankshaft is to be inserted into the hole of the bearing in SolidWorks, as shown in Figure 9. The bearing is fixed. The crankshaft is assembled to the bearing using two placement constraints, Mate: Concentric (Insert in Pro/ ENGINEER) and Mate: Coincident (Mate Surfaces in Pro/ENGINEER). The Mate: Concentric placement constraint eliminates two translational dof's and two rotational dof's. The Mate: Coincident constraint eliminates one translational dof's and two rotational dof's. As a result, only one dof, Rz, is left. SolidWorks allows designers to move (rotate) the crankshaft, by simply dragging the solid model, accordingly to the free dof. The designer is able to check the kinematics of the product in the assembly mode. This is a very attractive
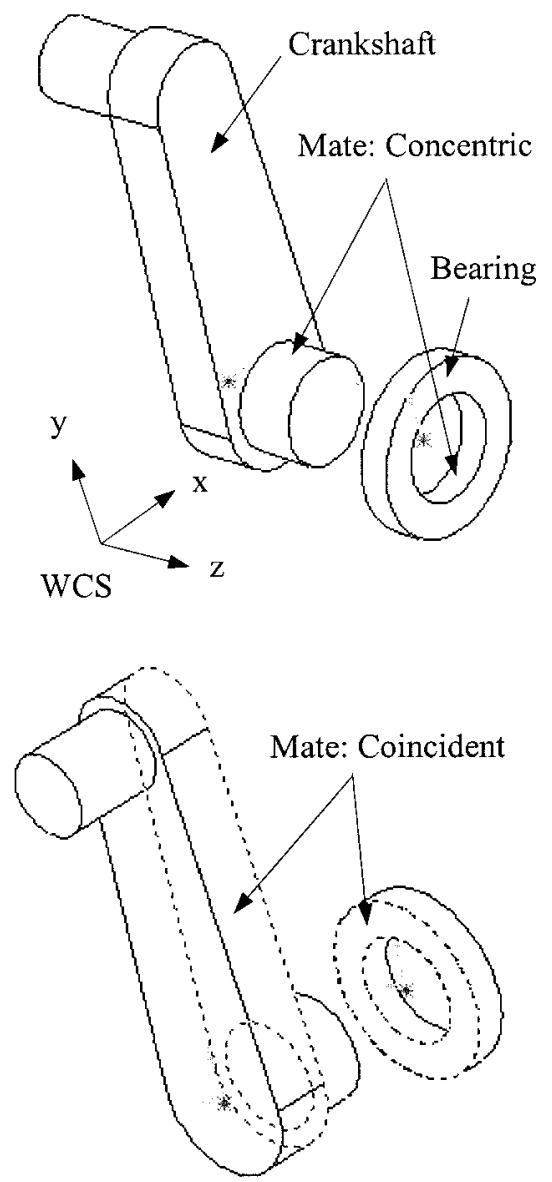

Figure 9. Bearing and crankshaft assembly. capability in mechanism design. In Pro/ENGINEER, such a rotational dof is allowed undefined.

The mathematical formulation and solution for assembly has been well documented, for example, in [2]. Note that, in some cases, Pro/ENGINEER and SolidWorks will not accept the constraints defined, if they are conflicting to existing ones or they over-constrain the part.

\subsection{The Independence Axiom}

Similar to part, the DIs in assembly can be uncoupled, coupled, and decoupled. The uncoupled design is again always superior to others. However, uncoupled DIs may not be always possible in practical applications, as illustrated earlier, using the slider-crank example with two coupled DIs. In general, it is required that the designer decouple the coupled DIs by adding DVs that relief the coupling effect, as discussed earlier. When the design is decoupled, design trade-off can be conducted to search for a near-optimal design following a systematic design method [4].

\subsection{The Information Axiom}

The information axiom at the assembly level can be addressed by adding relations or equations for dimensions across parts. For example, the diameter of a shaft must be related to that of the hole it inserts into, to reduce the number of DVs and capture the DIs. Note that, at the assembly level, in addition to complying with the two axioms, placements constraints and datum features must be properly defined to capture DIs.

\subsection{Slider-Crank Assembly in Pro/ENGINEER}

At the assembly level, the intent is to orient the crankshaft vertically and align the piston and piston pin horizontally with the center point of the lower semicircle of the crankshaft, as shown in Figure 4. Three assembly datum planes, $A D T M 1,2$, and 3, are created first. The crankshaft is assembled by properly aligning its datum planes with the assembly datum planes for a vertical orientation, as shown in Figure 10a. In order to assemble the rod, two additional datum planes are created in the assembly. ADTM4 is created by offsetting $A D T M 2$ three units upward (see Figure 10a). The datum plane ADTM5 is created by rotating ADTM4 with an angle $\mathrm{d} 1: 1=\sin ^{-1}(3 / 8)$. Note that $A D T M 5$ will be used to orient the rod. The rod is assembled to the crankshaft by three placement constraints: axis alignment, surface mate, and surface alignment, as shown in Figure 11.

In addition, the vertical position of ADTM4 and the rotation angle of ADTM5, which determine the configuration of the assembly, will be related to the crankshaft 


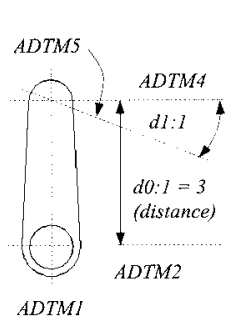

(a) Crankshaft

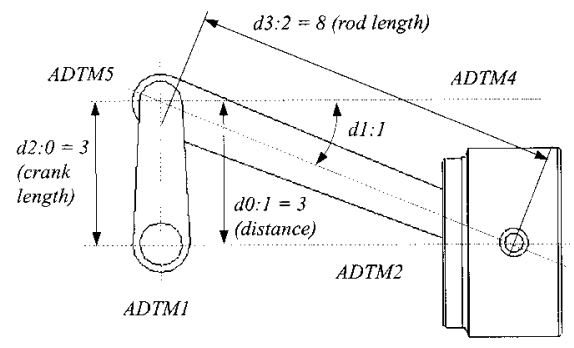

(b) Assembled Slider-Crank Mechanism
Figure 10. Parameter relations.

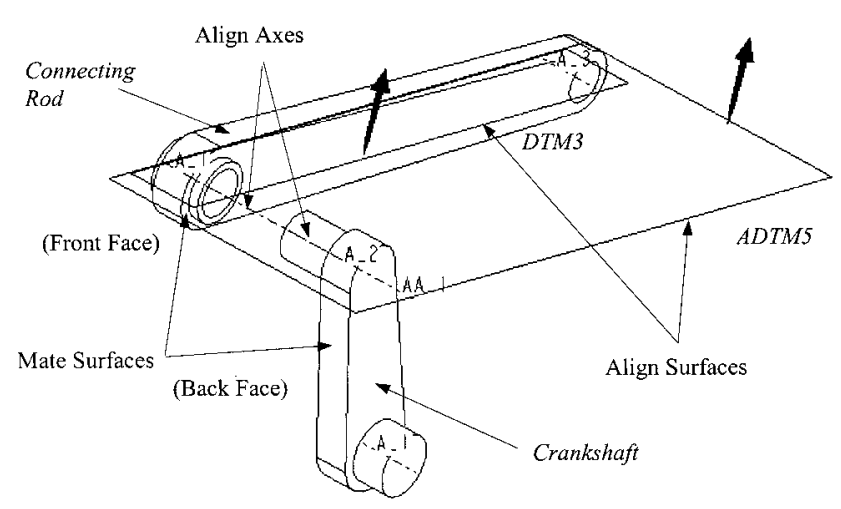

Figure 11. Assembly placement constraints defined for rod.

and rod lengths through two equations, $\mathrm{d} 0: 1=\mathrm{d} 2: 0$ and $\mathrm{d} 1: 1=\sin ^{-1}(\mathrm{~d} 2: 0 / \mathrm{d} 3: 2)$. Note that the first equation defines a relation that moves the datum plane ADTM4 up or down according to the crank length. The second equation defines the trigonometric relation of angle $\mathrm{d} 1: 1$ to the design variables $\mathrm{d} 2: 0$ (crank length) and $\mathrm{d} 3: 2$ (rod length). Dimension d $1: 1$ actually rotates ADTM5 according to the changes of $\mathrm{d} 2: 0$ and $\mathrm{d} 3: 2$. This is how the slider-crank mechanism can be parameterized. These equations define two independent design variables, i.e., $\mathrm{d} 2: 0$ and $\mathrm{d} 3: 2$, by relating four dimensions in assembly. Therefore, the information contents of the first design intent is reduced, hence complying with Axiom 2.

\subsection{Slider-Crank Assembly in SolidWorks}

The slider-crank mechanism is assembled in SolidWorks in a slightly different way. Since one of the objectives in SolidWorks assembly is to conduct kinematic analysis of the mechanism, a bearing part is introduced and is fixed in the assembly, as shown in Figure 12. Moreover, no additional datum plane is needed to orient the rod since its orientation will be determined by SolidWorks when the crankshaft rotates.

The crankshaft is assembled to bearing using Concentric and Coincident constraints, leaving one rotational dof, as shown in Figure 12. The connecting rod and piston pin are assembled following the similar way, also leaving one rotational dof for each part

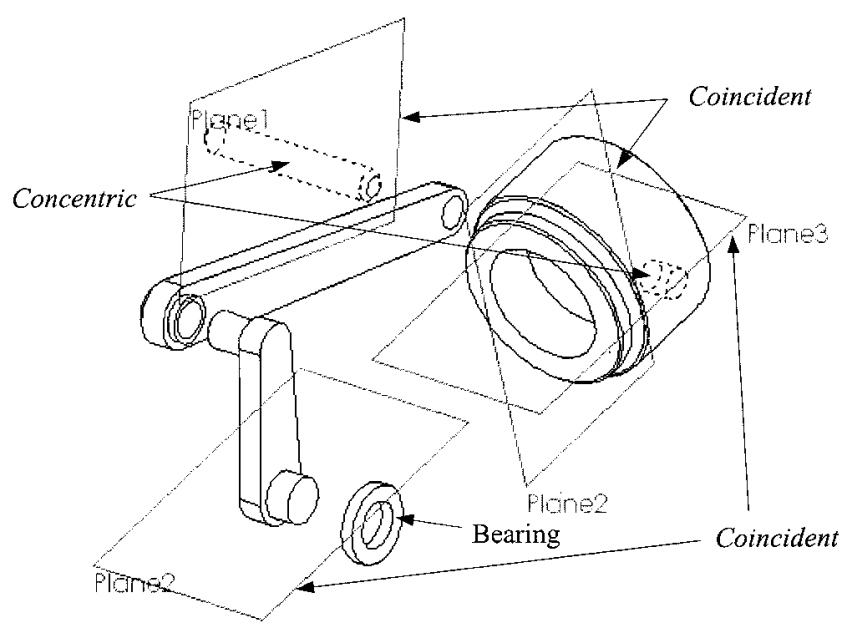

Figure 12. Placement constraints defined for the piston.

assembled. The piston is assembled using one Concentric and two Coincident constraints, as shown in Figure 12. Note that the second Coincident constraint that coincides Plane 3 of the piston and Plane 2 (horizontal plane) of the assembly confines the movement of the piston horizontally. When the length of the crankshaft or rod is changed, the assembly will be rebuilt, as shown in Figure 13, according to the trigonometric equation (see Figure 13d) with the distance between the piston and the crankshaft fixed temporarily, i.e.,

$$
\alpha=\sin ^{-1}\left(\frac{d 2: 0^{2}+d^{2}-d 3: 2^{2}}{2 \times d 2: 0 \times d}\right) .
$$

\subsection{Guidelines for Design Parameterization}

Based on the previous discussions, a set of guidelines for assembly is proposed in Table 2. Note that a build plan is again recommended before assembling any parts.

\section{Conclusions and Future Work}

In this paper, solid modeling and assembly techniques implemented in two major CAD tools, Pro/ENGINEER and SolidWorks, have been presented. A set of guidelines has been developed and proposed for the designers to parameterize the solid models at both part and assembly levels. These guidelines will facilitate the crossfunctional team to conduct product design in the CDM environment. A number of examples, including a slidercrank mechanism and a single piston airplane engine, have been presented to illustrate and demonstrate the validity and usefulness of the guidelines.

Although the initial set of guidelines have been demonstrated to be useful, they may not have covered every aspect in product design parameterization since 


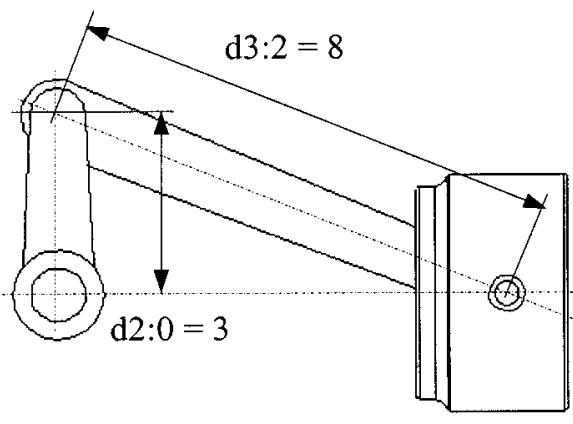

(a) Design Variables d2:0 and d3:2

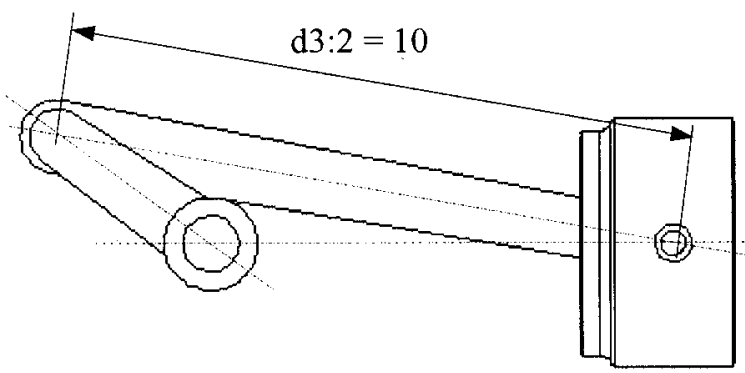

(c) d3:2 Changes to 10

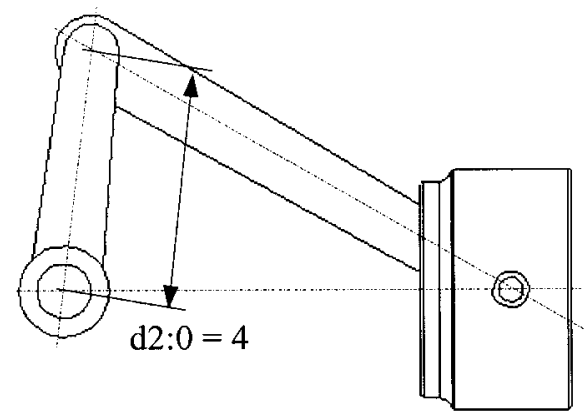

(b) d2:0 Changes to 4

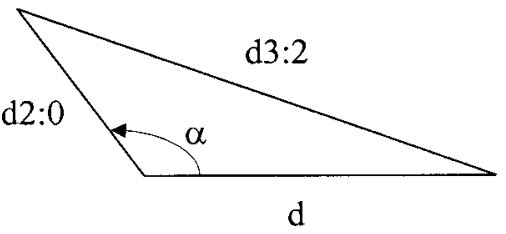

(d) Assembly Triangle

Figure 13. Change of length design variables in SolidWorks.

Table 2. Proposed guidelines for assembly parameterization.

\begin{tabular}{lll}
\hline & \multicolumn{1}{c}{ Independence Axiom } & \multicolumn{1}{c}{ Information Axiom } \\
\hline Datum & $\begin{array}{l}\text { A solid model should always be started with the } \\
\text { creation of three orthogonal datum planes. }\end{array}$ & $\begin{array}{l}\text { Additional datum features should be referenced to the indepen- } \\
\text { dent orthogonal datum planes. }\end{array}$ \\
Placement constraints & $\begin{array}{l}\text { Parts should be assembled together such that } \\
\text { changing the value of their DVs does not affect }\end{array}$ & $\begin{array}{l}\text { Eliminate all dof's except the dof needed for kinematic analysis. } \\
\text { their position in the assembly, unless the } \\
\text { position is a DV. }\end{array}$
\end{tabular}

limited examples are tested. More study is underway to include more realistic parts so that the parameterization guidelines can be more complete. At the same time, more industry standard CAD tools, such as CATIA, Unigraphics, and Mechanical Desktop, are being investigated.

\section{References}

1. Lee, K. (1999). Principles of $C A D / C A M / C A E$ Systems. Addison Wesley Longman, Inc. ISBN: 0-201-38036-6.

2. Zeid, I. (1991). CAD/CAM Theory and Practice. McGrawHill, Inc. ISBN 0-07-072857-7.

3. Chang, K.H., Silva, J. and Bryant, I. (December 1999). Concurrent design and manufacturing for mechanical systems. Concurrent Engineering Research and Applications ( CERA) Journal, 7(4): 290-308.
4. Tsai, C.S., Chang, K.H. and Wang, J. (August 1995). Integration infrastructure for a simulation-based design environment. Computers in engineering conference and the engineering data symposium. ASME Design Theory and Methodology Conference. Boston, M.A. pp. 9-20.

5. Chang, K.H., Choi, K.K., Wang, J., Tsai, C.S. and Hardee, E. (June 1998). A multi-level product model for simulation-based design of mechanical systems. Concurrent Engineering Research and Application ( CERA) Journal, 6(2): 131-144.

6. Suh, N.P. (1990). The Principles of Design, Oxford Series on Advanced Manufacturing. New York, NY: Oxford University Press.

7. Motenson, M.E. (1997). Geometric Modeling. 2nd Edn. NY: John Wiley \& Sons, Inc. ISBN 0-471-12957-7.

8. McMahon, C. and Browne, J. (1998). CADCAM. 2nd Edn. Addison-Wesley. ISBN 0-201-17819-2.

9. Toogood, R. (2000). Pro/ENGINEER Advanced Tutorial releases 2000i2. Schroff Development Co. ISBN 1-58503052-X. 


\section{Dr. Chang}

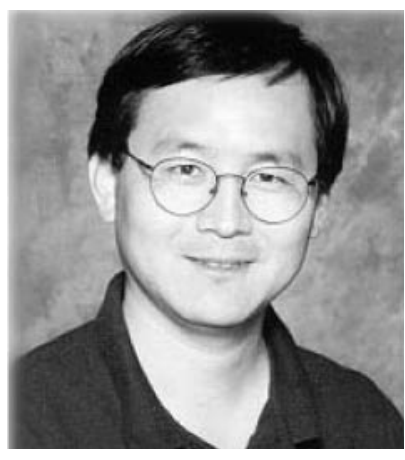

Dr. Chang is an Associate Professor in the School of Aerospace and Mechanical Engineering (AME) at the University of Oklahoma (OU). He received a B.S. in Mechanical Engineering from Taipei Institute of Technology, Taiwan, in 1980; a MS and PhD in Mechanical Engineering from The University of Iowa in 1987 and 1990, respectively. Since then, he joined the Center for Computer-Aided Design (CCAD) at Iowa as Research Scientist and CAE Technical Manager. In 1996, he joined Northern Illinois University as an Assistant Professor. In 1997, he joined OU. His areas of interest include Mechanical Fatigue and Reliability Analysis; Tools Integration for Concurrent Design and Manufacturing; Design Sensitivity Analysis and Optimization; Solid Freeform Fabrication, and Internet-Based CAD/
CAE/CAM using engineering multimedia, VRML and Java.

Javier J. Silva

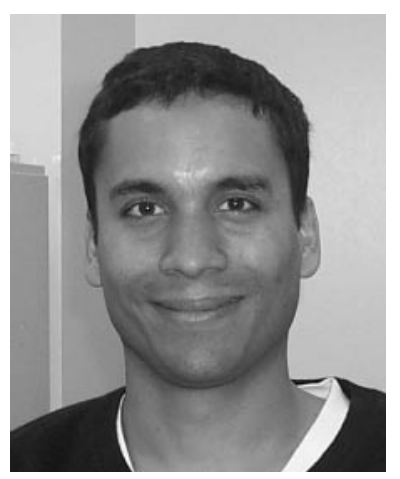

Javier J. Silva is a Graduate Research Assistant in the School of Aerospace and Mechanical Engineering (AME) at the University of Oklahoma (OU). He received a BS from the Colorado School of Mines in 1995, and he is currently pursuing his MS at OU sponsored by a Fulbright

Grant. He worked at Baker Hughes Inteq (Venezuela) from 1996 to 1998 as a Staff Engineer. His areas of interest are Concurrent Design and Manufacturing, use of CAD/CAM tools in Capturing the Design Intent, Design Optimization, and Rapid Prototyping. 